\title{
A FURTHER STUDY OF AN APPROXIMATION FOR LAST-EXIT AND FIRST-PASSAGE PROBABILITIES OF A RANDOM WALK ${ }^{1}$
}

\author{
D.J. DALEY \\ Australian National University \\ School of Mathematical Sciences \\ Centre for Mathematics and its Applications \\ A.C.T. 0200, AUSTRALIA \\ L.D. SERVI \\ GTE Laboratories Incorporated \\ 40 Sylvan Road \\ Waltham, MA 02254, USA
}

(Received April, 1994; revised July, 1994)

\begin{abstract}
Identities between first-passage or last-exit probabilities and unrestricted transition probabilities that hold for left- or right-continuous lattice-valued random walks form the basis of an intuitively based approximation that is demonstrated by computation to hold for certain random walks without either the left- or right-continuity properties. The argument centers on the use of ladder variables; the identities are known to hold asymptotically from work of Iglehart leading to Brownian meanders.
\end{abstract}

Key words: Random Walk, M/D/1 and D/M/1 Queues, Taboo Probabilities, Ladder Variables, First-passage Times, Last-exit Times.

AMS (MOS) subject classifications: Primary：60J15; Secondary：60K25.

\section{Introduction}

In Daley and Servi [1] some computations of conditional probabilities of a lattice-valued random walk arising from investigating the busy period in certain queueing models suggested that, in the contexts considered there, the approximation

$$
{ }_{0} p_{0 j}^{n} \equiv{ }_{(-\infty, 0]} p_{0 j}^{n} \equiv \mathbf{R}_{-} p_{0 j}^{n} \approx \alpha \frac{j}{n} p_{0 j}^{n} \quad(j=1,2, \ldots)
$$

of the last-exit probability of a random walk $\left\{X_{n}: n=0,1, \ldots\right\}$ on $\{0, \pm 1, \ldots\}$ with $X_{0}=0$, is a reasonable one, for $n$ not too small and some constant $\alpha$ that depends on the step-length distribution of the particular random walk. This note states more clearly the nature of the

\footnotetext{
${ }^{1}$ Work begun while visiting École Polytéchnique Fédérale de Lausanne and completed with support from GTE Laboratories Incorporated.
}

Printed in the U.S.A. (C)1994 by North Atlantic Science Publishing Company 
approximation in terms of ladder variables, studies it more fully for the last-exit probabilities considered in that previous paper, and also studies a similar approximation for a first-passage probability for which the heuristic justification is less appealing. Section 4 provides another probabilistic interpretation for $\alpha$, and thereby relates $\alpha$ to asymptotic results due to Spitzer [5].

The approximation is also suggested by limit results. Closer inspection of Iglehart [4] than noted in [1] shows that (1.1) is consistent with asymptotic properties that he proved involving Brownian meander. Specifically, he showed that under the assumption that the random walk has zero mean and finite third absolute moment, the conditioned process underlying the taboo probability on the left-hand side of (1.1) converges weakly to Brownian meander, while it is well known (the local limit theorem for zero-mean lattice-valued random walk with finite third moment) that the probability on the right-hand side converges (for $n \rightarrow \infty$ and $j \rightarrow \infty$ such that $j / \sqrt{n} \rightarrow y>0)$ to a normal density function, so the limit of the right-hand side is proportional to a density for Brownian meander.

\section{Approximating Last-exit Probabilities}

We study random walks $\left\{X_{n}\right\}$ satisfying $X_{n}=X_{n-1}+Y_{n}(n=1,2, \ldots)$ for independent identically distributed (i.i.d.) integer-valued random variables (r.v.s) $Y_{n}$ that are genuinely twosided so $\operatorname{Pr}\left\{Y_{n}<0\right\}>0$ and $\operatorname{Pr}\left\{Y_{n}>0\right\}>0$. We write

$$
a_{i}=\operatorname{Pr}\left\{Y_{n}=i\right\} \quad(i=0, \pm 1, \ldots) .
$$

Feller's ladder variable argument establishes that for right-continuous random walks (i.e., walks for which $\left.a_{i}=0 \quad(i=2,3, \ldots)\right)$, the last-exit probabilities ${ }_{0} p_{0 j}^{n}$ satisfy

$$
{ }_{0} p_{0 j}^{n}=\frac{j}{n} p_{0 j}^{n} \quad(j=1, \ldots, n ; n=1,2, \ldots) .
$$

In brief, for a sample path of $n$ steps of a right-continuous random walk from 0 to $j(j>0)$ with the walk always positive apart from its starting point, exactly $j$ of the steps leave points that are never revisited, and hence, exactly $j$ of the $n$ cycle permutations of the realized $n$ steps have the first step as a last-exit time from 0. We gave this argument in [1] as motivation for (1.1), and suggested below (2.6) there that (1.1) is a reasonable approximation for any random walk, at least for $j$ and $n$ not too small (inspection of its use in [1] shows that it appears reasonable, in the sense of being used where it matters, for $j=O(\sqrt{n})$ ). We now exhibit the nature of the approximation in more detail than earlier, and later plot both ${ }_{0} p_{0 j}^{n}$ and the ratio of the two sides in $(2.2)$,

$$
\alpha_{j, n} \equiv{ }_{0} p_{0 j}^{n} / \frac{j}{n} p_{0 j}^{n}
$$

for a particular non-trivial step-length distribution $\left\{a_{i}\right\}$ of interest.

Given any sample path with steps $\left\{Y_{1}, \ldots, Y_{n}\right\}$ leading to $\left(X_{0}, X_{n}\right)=(0, j)$, with $j>0$, there is always at least one cyclic rearrangement of these $n$ steps, $\left\{Y_{s+1}, \ldots, Y_{n}, Y_{1}, \ldots, Y_{s}\right\} \equiv\left\{Y_{1}^{\prime}, \ldots\right.$, $\left.Y_{n}^{\prime}\right\}$ say, for which the resulting path $\left\{X_{t}^{\prime}\right\}$ say, satisfies $X_{0}^{\prime}=X_{0}, X_{t}^{\prime}=X_{t-1}^{\prime}+Y_{t}^{\prime}(t=1, \ldots, n)$ and which, apart from $t=0$, is always positive, i.e.,

$$
X_{t}^{\prime}>X_{0}^{\prime}=0 \quad(t=1, \ldots, n) .
$$

For any such cyclic rearrangement there is a positive integer $r$ in $\{1, \ldots, \min (j, n)\}$ characterized as the number of last-exit epochs $t_{1}=0<t_{2}<\ldots<t_{r}<n$ for which 


$$
X_{t}^{\prime}>X_{t_{u}}^{\prime} \quad\left(\text { all } t>t_{u}, \text { each } u=1, \ldots, r\right) .
$$

If now the $\left\{Y_{1}^{\prime}, \ldots, Y_{n}^{\prime}\right\}$ are cyclically permuted by a shift $t_{u}$, we again obtain a rearrangement satisfying (2.4) and, for a possibly different set of epochs, (2.5). Thus there are exactly $r$ cyclic permutations of the steps satisfying (2.4), and for any sample path with $\left(X_{0}, X_{n}\right)=(0, j)$, this positive integer $r$ is a characteristic of the path. These are simple combinatorial facts that are another way of saying that the set of all sample paths with $\left(X_{0}, X_{n}\right)=(0, j)$ can be expressed as the union of $\min (j, n)$ disjoint, albeit not necessarily nonempty, sets, characterized by the possible numbers $r=1, \ldots, \min (j, n)$ of ladder indices that can occur in the cyclic rearrangements. Write $p_{0 j}^{n ; r}$ and ${ }_{0} p_{0 j}^{n ; r}$ for the probabilities associated with the sets of such sample paths, the former being the set of all paths with $r$ ladder indices, and the latter (taboo probabilities) with the paths satisfying (2.5). Since these decompositions are mutually exclusive and exhaustive it follows that

$$
{ }_{0} p_{0 j}^{n}=\sum_{r=1}^{\min (j, n)}{ }_{0} p_{0 j}^{n ; r} \quad \text { and } \quad p_{0 j}^{n}=\sum_{r=1}^{\min (j, n)} p_{0 j}^{n ; r},
$$

and by Feller's ladder variable argument,

$$
{ }_{0} p_{0 j}^{n ; r}=\frac{r}{n} p_{0 j}^{n ; r} .
$$

Then

$$
\begin{gathered}
{ }_{0} p_{0 j}^{n}=\sum_{r=1}^{\min (j, n)} \frac{r}{j} \cdot \frac{j}{n} p_{0 j}^{n ; r}=\frac{\sum_{r=1}^{\min (j, n)}(r / j) p_{0 j}^{n ; r}}{\sum_{r=1}^{\min (j, n)} p_{0 j}^{n ; r}} \cdot \frac{j}{n} p_{0 j}^{n} \\
=\alpha_{j, n} \frac{j}{n} p_{0 j}^{n}, \quad \approx \alpha \frac{j}{n} p_{0 j}^{n},
\end{gathered}
$$

showing that $0<\alpha_{j, n}<1$, with $\alpha_{j, n}=1$ and equality here when both sides are positive if and only if either $j=1$ or the walk is right-continuous. It was suggested below (2.6) in [1] that these quantities $\alpha_{j, n}$ should be approximately independent of $j$ and $n$ for those pairs $(j, n)$ for which $(j / n) p_{0 j}^{n}$ is not too far removed from its 'mode', which should cover those cases occurring in practice, when the distribution $\left\{a_{i}\right\}$ is unimodal. We now investigate this suggestion further by computing $\alpha_{j, n}$. For a whole family of conjugate distributions

$$
\left\{\frac{a_{i} R^{i}}{\sum_{j} a_{j} R^{j}}\right\}
$$

indexed by $R$ in $0<R<\infty$, subject to the convergence of the sum in the denominator in (2.9), this ratio is in fact independent of $R$ (cf. below equation (4.10) in [1]). Choosing that $R$ which gives the distribution a zero mean optimizes the computation, though, being independent of $R$, it is independent of whether the mean is zero or not. Later, where we use a local limit theorem, we require that the particular conjugate distribution (with zero mean) be used, so we assume hereafter that the one-step r.v.s $Y_{r}$ have zero mean.

Figure 1 plots both the taboo probabilities ${ }_{0} p_{0 j}^{n}$ and the ratios $\alpha_{j, n}$ for the cases $n=10,40$ in the case of the random walk with $\left\{a_{1+i}\right\}$ equal to a Poisson distribution with mean 1 (this corresponds to an $\mathrm{M} / \mathrm{D} / 1$ queue with traffic intensity 1). The taboo probabilities have in fact been multiplied by $4 n$ so as both to make them of comparable size and to make it easier to see the range of values of $j$ where the bulk of the probability mass is located, namely, for values of $j$ 
where $\alpha_{j, n} \approx \alpha=$ the limit of the 'average' values

$$
\alpha_{a v e}^{n} \equiv \frac{\sum_{j=1}^{\infty}{ }_{0} p_{0 j}^{n}}{\sum_{j=1}^{\infty}(j / n) p_{0 j}^{n}}
$$

(see the next paragraph); note the choice of scaling of the abscissae. The figure shows that for such $n$ the values of $\alpha_{j, n}$ in the region of the modal values of the taboo probability distributions change relatively slowly (and also approximately linearly), centered (roughly speaking) on the limiting value $\alpha$, and that the mode for the distribution of the taboo probabilities occurs where $j \approx \sqrt{n}$.

Note that, here and later, 'average' values of ratios like $\alpha_{j, n}$ are computed using the denominator like $(j / n) p_{0 j}^{n}$ as weights. This is equivalent to summing the numerators and denominators of (2.3) and defining $\alpha_{a v e}^{n}$ as at (2.10).

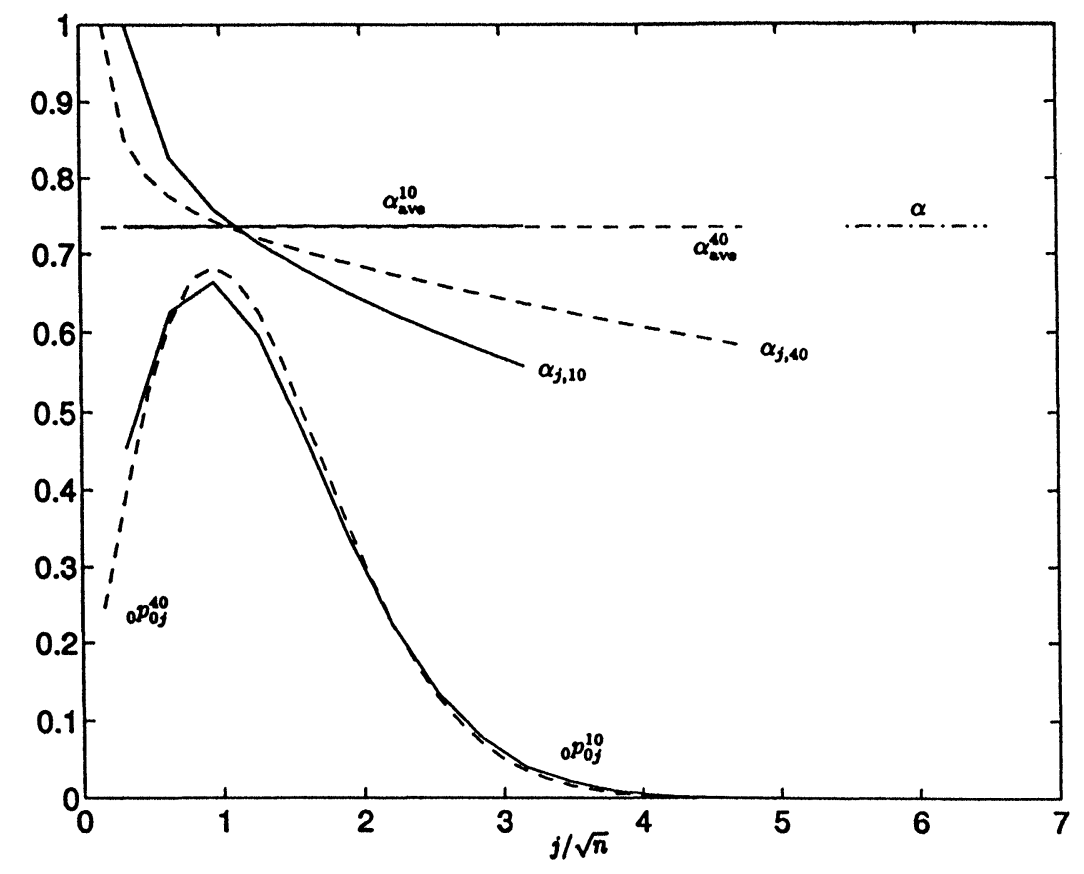

Figure 1. Rescaled taboo probabilities ${ }_{0} p_{0 j}^{n}$, probability ratios $\alpha_{j, n} \equiv \frac{{ }_{0} p_{0 j}^{n}}{(j / n) p_{0 j}^{n}}$, and average values $\alpha_{a v e}^{n}$ for left-continuous random walk with Poisson distributed step lengths.

Equation (2.8) shows that $j \alpha_{j, n}$ equals the average number of ladder steps between 0 and $j$, i.e., $\alpha_{j, n}=\mathrm{E} R_{j}^{n} / j$ where $R_{j}^{n}$ is the number of ascending ladder variables in the path of a random walk that is at $j$ after $n$ steps starting from 0 . If $\mathrm{E}(L)$ denotes the average length of ladder steps, then the elementary renewal theorem, if applicable, would assert that $\mathrm{E} R_{j}^{n} \approx j / \mathrm{E}(L)$, and hence

$$
\alpha_{j, n} \approx 1 / \mathrm{E}(L) .
$$


We return to this observation in Section 4.

\section{3. $\quad$ Approximating First-passage Probabilities}

We now study the first-passage probability

$$
(-\infty] p_{j,(-\infty, 0]}^{n} \equiv_{\mathbf{R}_{-}} p_{j, \mathbf{R}}^{n}=\operatorname{Pr}\left\{X_{n} \leq 0, X_{r}>0(r=1, \ldots, n-1) \mid X_{0}=j\right\},
$$

i.e., $\mathbf{R}_{-} p_{j, \mathbf{R}_{-}}^{n}$ is the probability that a first passage into the left half-line from $j>0$ occurs at time $n$. It was suggested in [1], with similar intuition as for (2.7), that the approximation

$$
\mathbf{R}_{-} p_{j, \mathbf{R}_{-}}^{n} \approx \beta \frac{j}{n} p_{j 0}^{n}
$$

should be reasonable under (imprecisely described) conditions as before. Recall that for leftcontinuous random walks, (3.2) holds for $\beta=1$ with equality because then $\mathbf{R}_{-} p_{j, \mathbf{R}_{-}}^{n}={ }_{\mathbf{R}_{-}} p_{j 0}^{n}$.

It is quickly apparent that the approximation suggested at (3.2) is more complex than (1.1). To see this, write

$$
\mathbf{R}_{-} p_{j, \mathbf{R}_{-}}^{n}=\sum_{k=0}^{\infty} \mathbf{R}_{-} p_{j,-k}^{n}
$$

this shows that the target state(s) concerned at time $n$ in (3.2) is a single state on the right-hand side but, except for left-continuous walks, more than one state on the left-hand side. At the very least then, we should be considering, not (3.2) but

$$
\mathbf{R}_{-} p_{j, \mathbf{R}_{-}}^{n} \approx \gamma \frac{j}{n} p_{j, \mathbf{R}_{-}}^{n}
$$

we study this numerically via the ratio

$$
\gamma_{j, n} \equiv \mathbf{R}_{-} p_{j, \mathbf{R}_{-}}^{n} / \frac{j}{n} p_{j, \mathbf{R}_{-}}^{n} \quad(j=1,2, \ldots, n)
$$

which, by analogy with the property $0<\alpha_{j, n} \leq 1$, we might expect to satisfy $0<\gamma_{j, n} \leq 1$.

To examine whether $\gamma_{j, n} \leq 1$ more generally than for left-continuous random walks, consider first $p_{j 0}^{n}$. For any sample path with $\left(X_{0}, X_{n}\right)=(j, 0)$, there is a characteristic number $r$ of cyclic rearrangements of the path for which the step to 0 at time $n$ is a first passage into $\mathbf{R}_{\text {_ }}$ from $X_{0}=j$. Then just as for $(2.6)$, we have

$$
\mathbf{R}_{-} p_{j 0}^{n}=\sum_{r=1}^{\min (j, n)} \mathbf{R}_{-} p_{j 0}^{n ; r} \quad \text { and } \quad p_{j 0}^{n}=\sum_{r=1}^{\min (j, n)} p_{j 0}^{n ; r}
$$

and

$$
\mathbf{R}_{-} p_{j 0}^{n ; r}=\frac{r}{n} p_{j 0}^{n ; r} \quad(r=1, \ldots, \min (j, n))
$$

again as before, at (2.7),

$$
\mathbf{R}_{-} p_{j 0}^{n}=\sum_{r=1}^{\min (j, n)} \frac{r}{j} \cdot \frac{j}{n} p_{j 0}^{n ; r} \equiv \beta_{j, n}^{\prime} \frac{j}{n} p_{j 0}^{n}
$$

for $0<\beta_{j, n}^{\prime} \leq 1$. 
The range of diagrams that can emerge from plotting $\left\{\beta_{j, n}^{\prime}\right\}$ and $\mathbf{R}_{-} p_{j 0}^{n}$ against $j$ is exactly the same as for the ratios $\left\{\alpha_{j, n}\right\}$ and the last-exit probabilities ${ }_{0} p_{0 j}^{n}$ as in Figure 1. To see this, define for given $n$ the random variables $\tilde{Y}_{r}=-Y_{n+1-r}$ and $\tilde{X}_{r}=\tilde{X}_{0}+\tilde{Y}_{1}+\ldots+\tilde{Y}_{r}(r=$ $1, \ldots, n), \tilde{X}_{0}$ being given. Then

and

$$
\begin{gathered}
p_{j 0}^{n}=\operatorname{Pr}\left\{X_{n}=0 \mid X_{0}=j\right\}=\operatorname{Pr}\left\{X_{n}=-j \mid X_{0}=0\right\} \\
=\operatorname{Pr}\left\{\tilde{X}_{n}=j \mid \tilde{X}_{0}=0\right\} \equiv \tilde{p}_{0 j}^{n} \text { say }
\end{gathered}
$$

$$
\begin{gathered}
\mathbf{R}_{-} p_{j 0}^{n}=\operatorname{Pr}\left\{X_{n}=0, X_{r}>0 \quad(r=1, \ldots, n-1) \mid X_{0}=j\right\} \\
\left.=\operatorname{Pr}\left\{X_{n}=-j, X_{r}>-j(r=1, \ldots, n-1)\right\} X_{0}=0\right\} \\
=\operatorname{Pr}\left\{\tilde{X}_{n}=j, \tilde{X}_{n+1-r}>0 \quad(r=1, \ldots, n-1) \mid \tilde{X}_{0}=0\right\} \equiv_{\mathbf{R}_{-}} \tilde{p}_{0 j}^{n} \text { say. }
\end{gathered}
$$

Then the ratio $\beta_{j, n}^{\prime}$ can equally arise from last-exit probabilities as asserted.

Now it is equally true that any sample path contributing to $\mathbf{R} p_{j,-k}^{n}$ for any $k$ in $\{0,1, \ldots\}$, has a first passage into $\mathbf{R}_{-}$at its last step and at most $j-1^{-}$downward first-passage steps amongst the times $1, \ldots, n-1$. Denoting the number of these downward first-passage steps by $r$, it need no longer be true, however, that all $r$ cyclic permutations of steps that contribute to $p_{j,-k}^{n ; r}$ say are simultaneously first passages into $\mathbf{R}_{-}$, because there is now the added requirement that the last step be of length $\geq 1+k$. Thus,

$$
\mathbf{R}_{-} p_{j,-k}^{n ; r} \leq \frac{r}{n} p_{j,-k}^{n ; r}
$$

equivalently, for some $0<\beta_{j, n ; k, r}^{\prime \prime} \leq 1$,

$$
\mathbf{R}_{-} p_{j,-k}^{n ; r}=\beta_{j, n ; k, r}^{\prime \prime} \frac{r}{j} \cdot \frac{j}{n} p_{j,-k}^{n ; r} \quad(r=1, \ldots, \min (j, n)) .
$$

Then,

$$
\begin{aligned}
\mathbf{R}_{-} p_{j, \mathbf{R}_{-}}^{n}= & \sum_{k=0}^{\infty} \sum_{r=1}^{\min (j, n)} \mathbf{R}_{-} p_{j,-k}^{n ; r}=\left[\sum_{k=0}^{\infty} \sum_{r=1}^{\min (j, n)} \beta_{j, n ; k, r}^{\prime \prime} \frac{r}{j} p_{j,-k}^{n ; r}\right] \frac{j}{n} \\
= & \frac{\sum_{k=0}^{\infty} \sum_{r=1}^{\min (j, n)} \beta_{j, n ; k, r}^{\prime \prime}(r / j) p_{j,-k}^{n ; r}}{\sum_{k=0}^{\infty} \sum_{r=1}^{\min (j, n)} p_{j,-k}^{n ; r}} p_{j, \mathbf{R}}^{n} \equiv \gamma_{j, n} \frac{j}{n} p_{j, \mathbf{R}}^{n}
\end{aligned}
$$

for some $0<\gamma_{j, n} \leq 1$.

We conclude therefore that a first-passage probability analogue of the last-exit probability approximation at (2.7) holds, albeit of the form (3.4). 


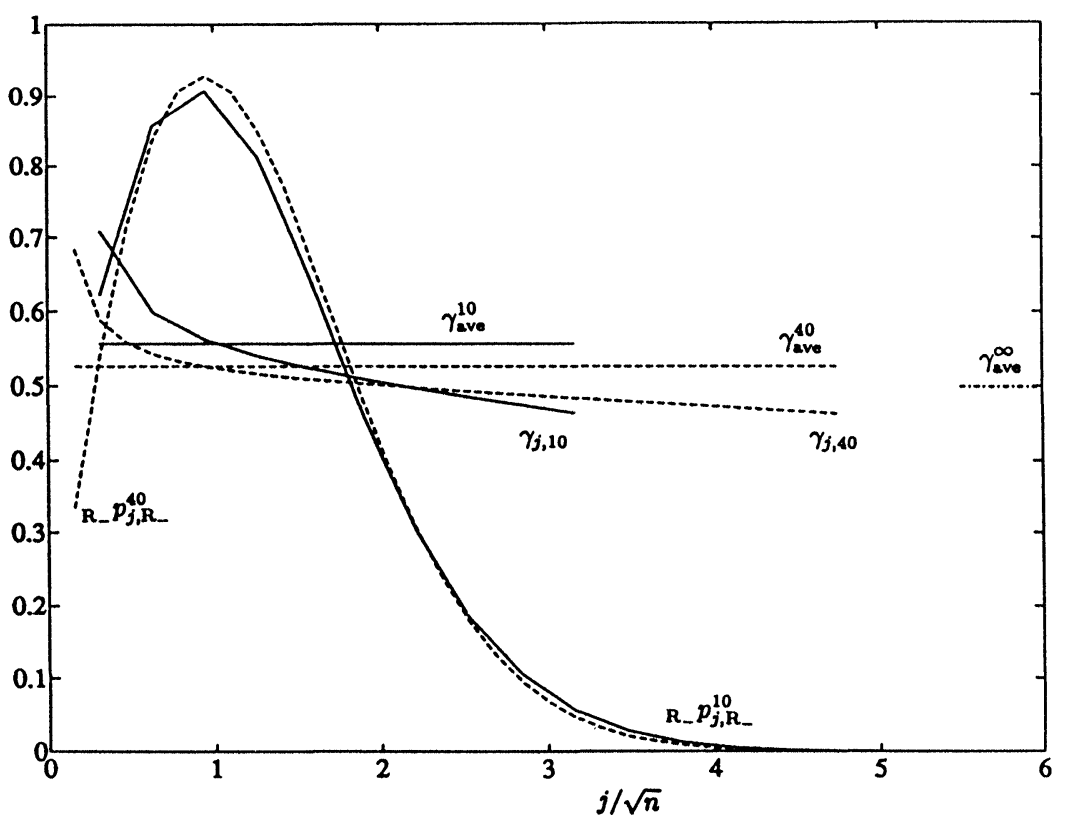

Figure 2. Rescaled taboo probabilities $\mathbf{R}_{-} p_{j, \mathbf{R}_{-}}^{n}$, probability ratios $\gamma_{j, n} \equiv \frac{\mathbf{R}_{-} p_{j, \mathbf{R}_{-}}^{n}}{(j / n) p_{j, \mathbf{R}_{-}}^{n}}$, and average values $\gamma_{a v e}^{n}$ for right-continuous random walk with Poisson distributed step lengths

Figure 2 plots both the taboo probabilities $\mathbf{R}_{-} p_{j, \mathbf{R}_{-}}^{n}$ and the ratios $\gamma_{j, n}$ for the cases $n=10,40$ in the case of a random walk with $\left\{a_{1-i}\right\}(i=1,0,-1, \ldots)$ a Poisson distribution with mean 1 (this corresponds to a $\mathrm{D} / \mathrm{M} / 1$ queue with traffic intensity 1). The taboo probabilities are rescaled by $4 n$ as for Figure 1, and the $x$-axis is similarly scaled. The comments concerning $\alpha_{j, n}$ there are applicable mutatis mutandis to $\gamma_{j, n}$ here. The only features that appear to us to be markedly different are firstly that the first-passage probabilities from 1 to $\mathbf{R}_{-}$ do not have a constant value for the ratio as for the last exit probabilities from 0 to 1 , and secondly that the 'average' values

$$
\gamma_{\text {ave }}^{n}=\frac{\sum_{j=1}^{\infty} \mathbf{R}_{-} p_{j, \mathbf{R}_{-}}^{n}}{\sum_{j=1}^{\infty}(j / n) p_{j, \mathbb{R}_{-}}^{n}}
$$

vary with $n$ much more than their $\alpha$ analogues.

Just as $\alpha_{j, n}$ has an interpretation as the reciprocal of the average length of ascending ladders steps (cf. equation (2.11)), equation (3.12) shows that $j \gamma_{j, n}$ equals the mean number of descending ladder epochs in a random walk starting from $j$ and entering $\mathbf{R}_{-}$for the first time after $n$ steps. The elementary renewal theorem now implies that $\gamma_{j, n}$ is approximately like the reciprocal of the average length of descending ladder steps.

Recall that for first-passage probabilities of a random walk we can write a forwards Chapman-Kolmogorov equation, namely, 


$$
\begin{aligned}
\mathbf{R}_{-} p_{j, \mathbf{R}_{-}}^{n} & =\sum_{i=0}^{\infty} \mathbf{R}_{-} p_{j,-i}^{n}=\sum_{k=1}^{\infty} \mathbf{R}_{-} p_{j k}^{n-1} \sum_{i=0}^{\infty} a_{-(k+i)} \\
& \approx \mathbf{R}_{-} p_{j 1}^{n-1} \sum_{k=1}^{\infty} \sum_{i=0}^{\infty} a_{-}(k+i) \\
& =\mathbf{R}_{-} p_{j 1}^{n-1} \sum_{\ell=1}^{\infty} \ell a_{-\ell}=\mathbf{R}_{-} p_{j 1}^{n-1} \mathrm{E} Y- \\
& =\mathbf{R}_{-} p_{j 1}^{n-1} \frac{1}{2} \mathrm{E}|Y|
\end{aligned}
$$

where $Y$ is a generic one-step random variable (recall that $\mathrm{E} Y=0$ so $\mathrm{E} Y{ }_{-}=\mathrm{E} Y{ }_{+}=\frac{1}{2} \mathrm{E}|Y|$ ). Similarly,

$$
\mathbf{R}_{-} p_{j 0}^{n}=\sum_{k=1}^{\infty} \mathbf{R}_{-} p_{j k}^{n-1} a_{-k} \approx \mathbf{R}_{-} p_{j 1}^{n-1} \operatorname{Pr}\{Y<0\}
$$

so since $Y$ is integer-valued

$$
\mathbf{R}_{-} p_{j \mathbf{R}_{-}}^{n} \approx \mathbf{R}_{-} p_{j 0}^{n} \frac{\mathrm{E} Y-}{\operatorname{Pr}\{Y<0\}}=\mathbf{R}_{-} p_{j 0}^{n} \mathrm{E}(|Y| \mid Y<0) \geq \mathbf{R}_{-} p_{j 0}^{n}
$$

Coupling (3.16) with (3.8) implies that we may anticipate that (3.2) holds provided that we identify $\beta$ as an 'average' value of

$$
\beta_{j, n} \equiv \frac{\mathbf{R}_{-} p_{j, \mathbf{R}}^{n}}{(j / n) p_{j 0}^{n}} \approx \beta_{j, n}^{\prime} \mathrm{E}(|Y| \mid Y<0),
$$

which need not be $<1$.

Figure 3 shows plots of $\left\{\beta_{j, n}\right\}$ and $\rho_{j, n} \equiv \mathbf{R}_{-} p_{j, \mathbf{R}_{-}}^{n} / \mathbf{R}_{-} p_{j 0}^{n}\left(\right.$ so, $\left.\rho_{j, n}=\beta_{j, n} / \beta_{j, n}^{\prime}\right)$, for the same distribution for $Y_{n}$ as in Figure 2. Note that for this distribution, $\mathrm{E}(|Y| \mid Y<0)=$ $e^{-1} /\left(1-2 e^{-1}\right)=1 /(e-2)=1.39221$. The plot of the former ratios shows much the same behavior as for the ratios in Figures 1 and 2. The latter indicates relatively little dependence on either $j$ or $n$, and that the $\rho_{j, n}$ are of the order of magnitude of the conditional expectation factor at (3.16) without being equal to it.

We conclude this section with an aside, discussing an approximation that is relevant for values of $j$ and $n$ where (3.2) may not hold. The difference between (3.2) and (3.4) lies in the right-hand side: in the former there is a density (with respect to counting measure), while the latter has a tail probability. Because both probabilities concern a large number of steps of a random walk, we can appeal to both local and the usual central limit theorem. Using $\Phi(\cdot)$ and $\varphi(\cdot)$ for the distribution and density functions respectively of a standard Gaussian random variable with zero mean and unit variance, and writing $\sigma$ for the standard deviation of the steps of the random walk, the right-hand side of $(3.4)$ is like $\gamma_{j, n}(j / n) \Phi(-j / \sigma \sqrt{n})$, and, under further 


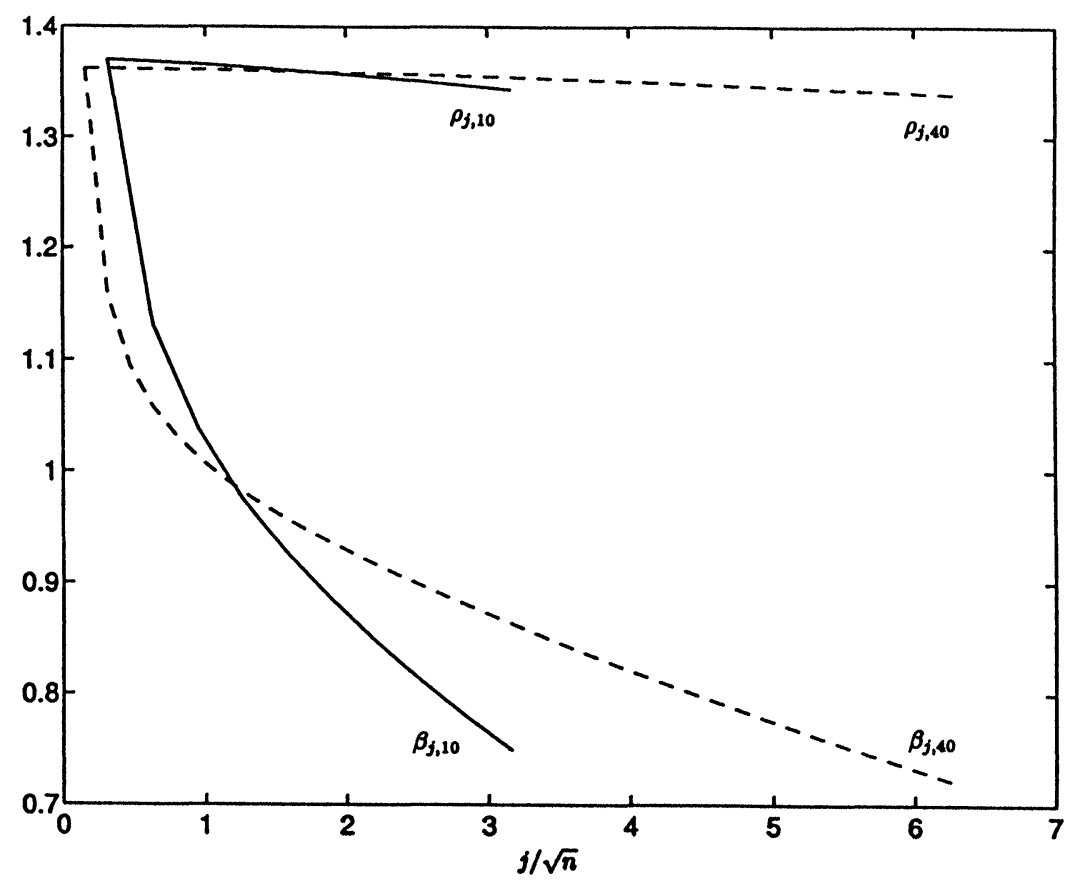

Figure 3. Probability ratios $\rho_{j, n} \equiv \frac{\mathbf{R}_{-} p_{j, \mathbf{R}_{-}}^{n}}{\mathbf{R}_{-} p_{j 0}^{n}}$ and $\beta_{n, n} \equiv \frac{\mathbf{R}_{-} p_{j, \mathbf{R}_{-}}^{n}}{(j / n) p_{j, \mathbf{R}_{-}}^{n}}$ for a right-continuous random walk with Poisson distributed step lengths, $n=10,40$.

conditions, the right-hand side of $(3.2)$ is like $\beta^{\prime}(j / n) \varphi(j / \sigma \sqrt{n}) / \sigma \sqrt{n}$. Mills' ratio asserts that for large positive $x, x \Phi(-x) / \varphi(x) \approx 1$, so (3.12) implies that for large $j / \sqrt{n}$,

$$
\begin{aligned}
\mathbf{R}_{-} p_{j, \mathbf{R}_{-}}^{n} & \approx \gamma_{j, n} \frac{j}{n} \Phi\left(-\frac{j}{\sigma \sqrt{n}}\right) \approx \gamma_{j, n} \frac{j}{n} \cdot \frac{\sigma \sqrt{n}}{j} \varphi\left(\frac{j}{\sigma \sqrt{n}}\right) \\
& =\gamma_{j, n} \frac{\sigma}{\sqrt{n}} \varphi\left(\frac{j}{\sigma \sqrt{n}}\right) \approx \gamma_{j, n} \sigma^{2} p_{j 0}^{n} .
\end{aligned}
$$

This is not of the from (3.2), but neither should we expect it, because (2.7) and (3.4), in the numerical cases we studied, hold in the sense that $\alpha$ and $\gamma$ are 'average' values of $\alpha_{j, n}$ and $\gamma_{j, n}$ that reflect these latter in the neighborhood of $j=O(\sqrt{n})$, where $j / \sqrt{n}$ is not necessarily large.

\section{Some Asymptotics}

Write $T$ for the first entrance time of $\left\{X_{n}\right\}$ into $\{0,-1, \ldots\}$, starting from $X_{0}=0$. Suppose it is true that

$$
\mathbf{R}_{-} p_{0 j}^{n} \approx \frac{\alpha j}{n} p_{0 j}^{n}
$$

at least for $n$ not small and $j \leq n$. Then addition of each side over $j$ gives 


$$
\operatorname{Pr}\{T>n\}=\sum_{j=1}^{\infty} \mathbf{R}_{-} p_{0 j}^{n} \approx \frac{\alpha}{n} \cdot \mathrm{E}\left(X_{n} ; X_{n}>0\right)=\frac{\alpha}{n} \cdot \frac{1}{2} \mathrm{E}\left(\left|X_{n}\right|\right)
$$

and now appealing to the central limit theorem and using the fact that $\frac{1}{2} \mathrm{E}(|Z|)=\sigma_{Z} / \sqrt{2 \pi}$ for a normally distributed random variable $Z$ with zero mean and standard deviation $\sigma_{Z}$ gives the second approximation below from $\sigma^{2}=\operatorname{var}(Y)$ :

$$
\operatorname{Pr}\{T>n\} \approx \frac{\alpha}{n} \cdot \mathrm{E}\left(\frac{1}{2}\left|X_{n}\right|\right) \approx \frac{\alpha}{n} \cdot \sigma \sqrt{\frac{n}{2 \pi}}=\frac{\alpha \sigma}{\sqrt{2 \pi n}} .
$$

Now from Theorem 3.5 of Spitzer [5], quoted at (2.1) of Iglehart [4] (but, we have not found it in Spitzer [6]), the left-hand side here is asymptotically like

$$
\frac{c}{\sqrt{\pi n}} \text { where } c=\exp \left(-\sum_{k=1}^{\infty} \frac{\frac{1}{2}-\operatorname{Pr}\left\{X_{k}>0\right\}}{k}\right)
$$

(as an aside, note from Proposition 18.8 of Spitzer [6] that for $T^{\prime} \equiv$ the first entrance time of $\left\{X_{n}\right\}$ into $\{1,2, \ldots\}, \operatorname{Pr}\left\{T^{\prime}>n\right\} \approx 1 /(c \sqrt{\pi n})$ ). Equation (4.3) with (4.4) implies that $\alpha$ can be given in terms of $c$, namely

$$
\alpha \approx \frac{\sqrt{2}}{\sigma} c
$$

Notice that this is indeed independent of $n$. Using the first approximation in (4.3) now gives

$$
\lim _{n \rightarrow \infty} \alpha_{n} \rightarrow \alpha \text { where } \alpha_{n} \equiv \frac{n \operatorname{Pr}\{T>n\}}{\mathrm{E}\left\{X_{n} ; X_{n}>0\right)}=\frac{n \sum_{j=1}^{\infty} \mathbf{R}_{-} p_{0 j}^{n}}{\sum_{j=1}^{\infty} j p_{0 j}^{n}}
$$

leading to the data in Table 1 for $Y_{n}+1$ Poisson-distributed with mean 1. (The term for $n=\infty$ comes from (4.4) used as described in Section 5.)

Comparison with part (a) of Proposition 18.5 of Spitzer [6] shows that our $\alpha$ is just the reciprocal of the mean of the step-length of the upward random walk of ascending ladder steps of $\left\{X_{n}\right\}$. This is exactly what follows from the discussion around (2.11).

TABLE 1

Values of $\alpha_{n}$ for Poisson distribution for steps

$\begin{array}{ccccccccccc}n & 5 & 10 & 15 & 20 & 30 & 40 & 50 & 60 & 70 & \infty \\ \alpha_{n} & 0.75660 & 0.73692 & 0.73486 & 0.73581 & 0.73508 & 0.73525 & 0.73535 & 0.73542 & 0.73547 & 0.73576\end{array}$

Inspection of the numerical value of $\alpha$ shown in Table 1 suggests that for this particular random walk, this $\alpha$ equals $\mathrm{E}(|Y|)=2 e^{-1}=0.735759$.

We have not been able to obtain similarly useful expressions for $\beta$ and $\gamma$ from the approximations of (3.2) and (3.4). Summation over $j$ of the variable quantities in the right-hand sides yields closed form expressions in terms of the first and second moments of the positive parts of $X_{n}$. Similar summation of the left-hand side yields only the tautology

$$
\sum_{j=1}^{\infty} \mathbf{R}_{-} p_{j \mathbf{R}_{-}}^{n}=\sum_{j=1}^{\infty} \operatorname{Pr}\left\{T_{j}=n\right\}
$$


where $T_{j}$ denotes a r.v. for the time of first passage into $\mathbf{R}_{-}$of a random walk starting at $j>0$.

\section{Computational Notes}

Using the asymptotics above as a guide, a practical route to the computation of $c$ is to evaluate

$$
C_{n} \equiv \sum_{k=1}^{n} \frac{\frac{1}{2}-\operatorname{Pr}\left\{S_{k}>0\right\}}{k}
$$

and, assuming the power series representation below holds, to write

$$
C_{n}=C_{\infty}+n^{-\frac{1}{2}}\left(a+\frac{b}{n}+O\left(n^{-2}\right)\right)
$$

for some constants $a$ and $b$. Then we can improve on $C_{n}$ as an approximation to $C_{\infty}=c$ by considering

$$
C_{\infty, n} \equiv 2 C_{4 n}-C_{n}=C_{\infty}-\frac{3}{4} \cdot \frac{b}{n \sqrt{n}}-O\left(n^{-5 / 2}\right)
$$

and neglecting terms that are $O\left(n^{-3 / 2}\right)$. Even better is to neglect terms only after $n^{-3 / 2}$, yielding

$$
C_{\infty, n}^{(2)} \equiv \frac{8 C_{\infty, 4 n}-C_{\infty, n}}{7}=C_{\infty}+O\left(n^{-5 / 2}\right)
$$

In the case of the Poisson distribution with mean 1 (i.e., the same example as underlies Figure 1), using Fortran 77 double precision arithmetic and evaluating the sum in the exponent of (4.4), gives e.g., $C_{256}=0.620219, C_{64}=0.587238$, so $C_{\infty, 64}=0.65320$. Further computation shows convergence of the improvement towards 0.653380 before underflow errors start influencing the computation. From this one would have $\alpha \approx 0.735793$. The second of the improvements converges and stabilizes (to six significant figures) to 0.653426 and hence $\alpha \approx 0.735759$, for $n$ between 528 and 752 before underflow errors have any influence.

For the same example, computing $\alpha$ directly gives values of $\alpha_{j, n}$ as sketched in Figure 1 for $n=10,40$, all normalized so as to be plotted against $j / \sqrt{n}$. Table 1 lists values of $\alpha_{n}$ that come from using (4.6) with these computations.

\section{Acknowledgement}

We thank Professor Hans Bühlmann, ETH Zurich, for asking us for details about $\alpha_{j, n}$.

\section{References}

[1] Daley, D.J. and Servi, L.D., Approximating last-exit probabilities of a random walk, with application to conditional queue length moments within busy periods of M/GI/1 queues, $J$. Appl. Probab. 31A (1994), (Takacs Festshrift) (to appear).

[2] Feller, W., On combinatorial methods in fluctuation theory, in: Probability and Statistics (The Harold Cramér Volume) (ed. Ulf Grenander), Almqvist \& Wiksell, Stokholm, and Wiley, New York 1959.

[3] Feller, W., An Introduction to Probability Theory and its Applications, Vol. 2 (2nd ed.), 
Wiley, New York 1971.

[4] Iglehart, D.L., Conditioned limit theorems for random walks, in: Stochastic Processes and Related Topics (ed. M.L. Puri), Vol. 1, Academic Press, New York 1975.

[5] Spitzer, F., A Tauberian theorem and its probability interpretation, Trans. Amer. Math. Soc. 94 (1960), 150-169.

[6] Spitzer, F., Principles of Random Walk, Van Nostrand, Princeton 1964. [2nd ed., Springer-Verlag, New York 1976]. 


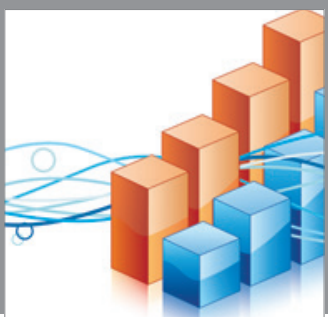

Advances in

Operations Research

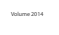

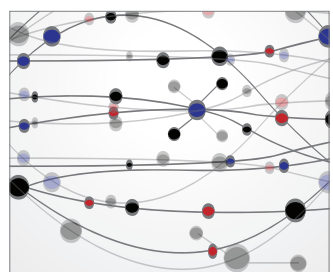

\section{The Scientific} World Journal
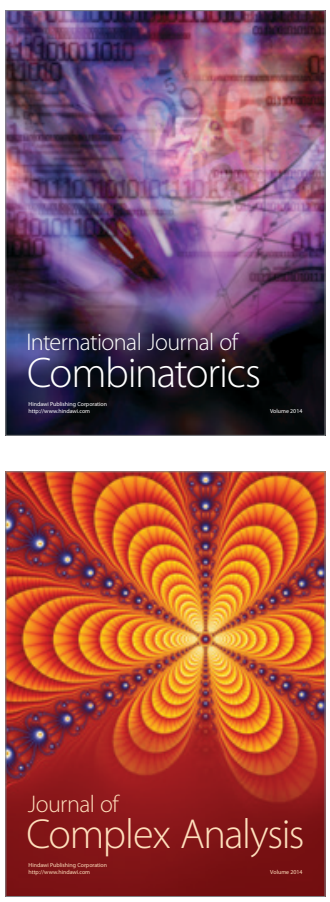

International Journal of

Mathematics and

Mathematical

Sciences
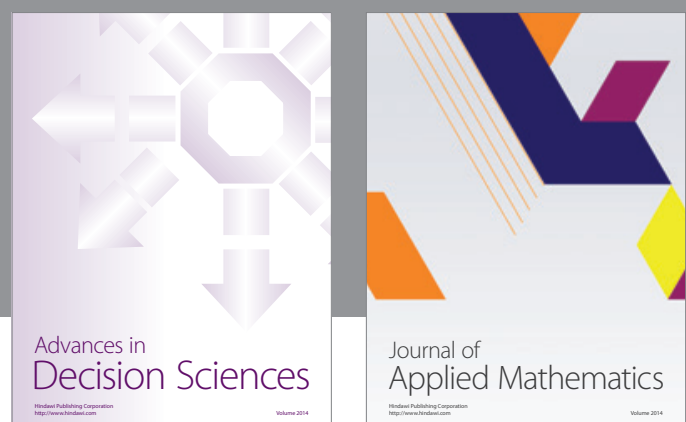

Journal of

Applied Mathematics
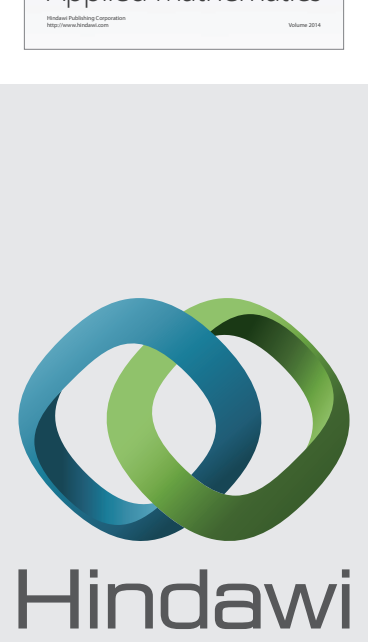

Submit your manuscripts at http://www.hindawi.com
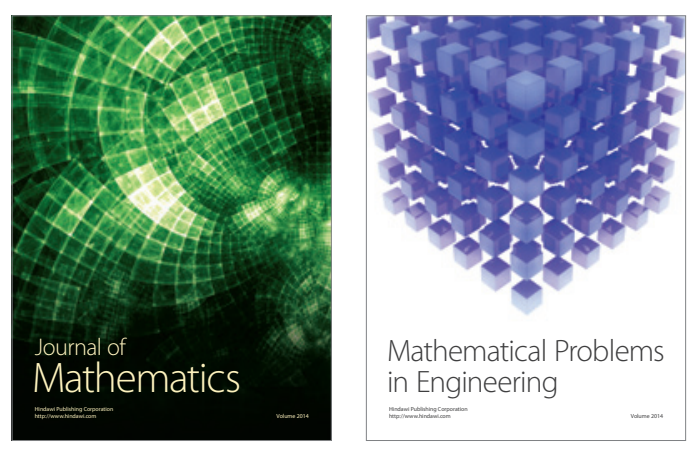

Mathematical Problems in Engineering
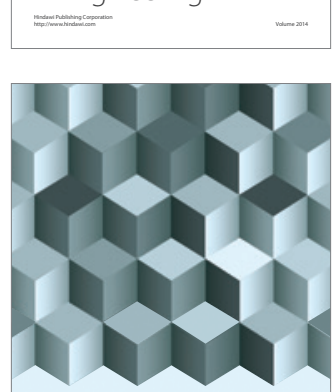

Journal of

Function Spaces
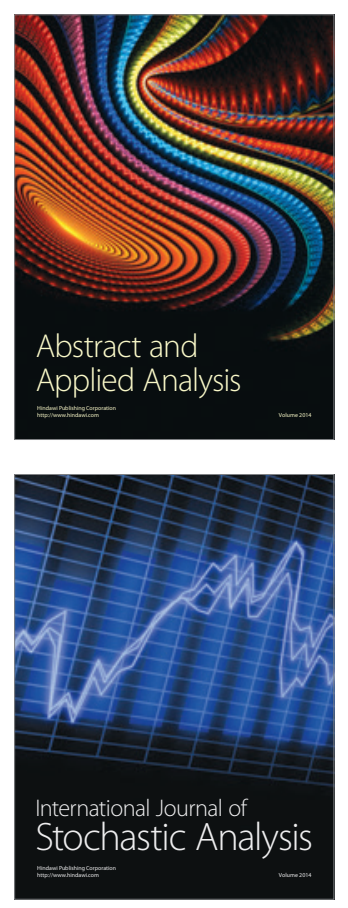

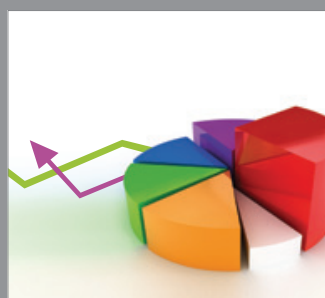

ournal of

Probability and Statistics

Promensencen
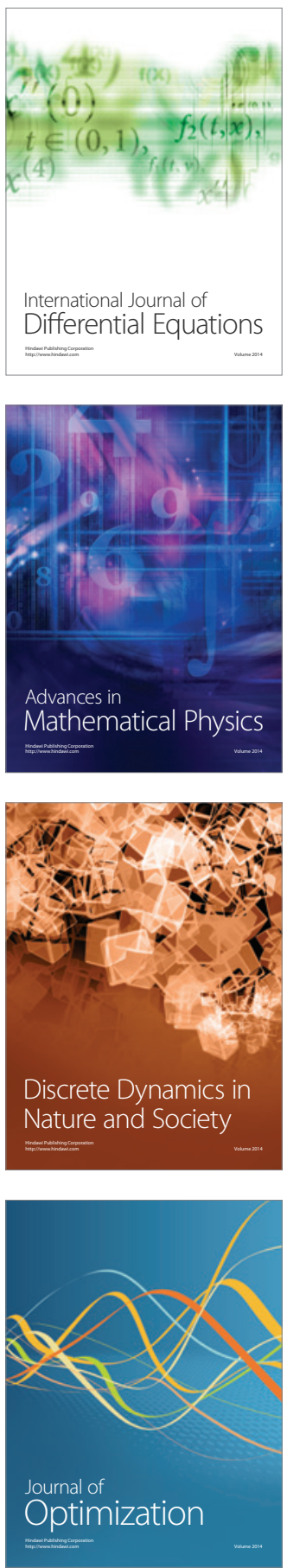